\title{
Out with the old, in with the new... strategies based on stem cells to design new models of alzheimer's disease
}

\begin{abstract}
Since Yamanaka's group successfully discovered iPSCs technology a decade ago, new approaches for disease modeling have happened in the field. iPSCs have become the primary alternative to ESCs and is considered as a potential tool for modeling neurodegenerative diseases. The most prevalent form of dementia worldwide is $\mathrm{AD}$, with aging being the major risk factor to develop it. Although it was described more than a hundred years ago, currently it doesn't exist a cure for this disorder, and ordinary treatments can only temporarily alleviate their symptoms. It has been reported that only $5 \%$ of $\mathrm{AD}$ cases are inherited, showing the remaining $95 \%$ a sporadic origin. Our understanding of $\mathrm{AD}$ pathogenesis is currently limited by difficulties in obtaining live neurons from patients and the limitations to model the sporadic form of the disease. Given that, iPSCs can be derived from somatic cells of AD patients and subsequently be differentiated into neurons, the development of therapies based on the use of stem cells might be a promising novel tool for cellular replacement therapy, in addition to disease modeling and drug discovery for AD. Direct reprogramming, cocktails of small molecules, gene editing or 3D cell culture are among those new strategies that are improving the application of stem cells in regenerative medicine. Here, we show how present approaches in the field of iPSCs can contribute to create novel AD disease models.
\end{abstract}

Keywords: alzheimer's disease, neurodegenerative disorder, disease models, induced pluripotent stem cells, induced neurons, genome editing, regenerative medicine
Volume 2 Issue 2 - 2017

\author{
Ana Revilla \\ Cell Biology Department, Universidad Complutense de Madrid, \\ Spain \\ Correspondence: Ana Revilla, Cell Biology Department, \\ Faculty of Medicine, Universidad Complutense de Madrid, Plaza \\ Ramón y Cajal s/n, Madrid, Spain, Tel 3491394I637, \\ Email anarevil@ucm.es
}

Received: January 22, 2017 | Published: February 24, 2017
Abbreviations: hESCs, human embryonic stem cells; iPSCs, induced pluripotent stem cells; NSCs, neural stem cells; iNSCs, induced neural stem cells; iNs, induced neurons; AD, alzheimer's disease; FAD, familial ad; SAD, sporadic ad; PSEN1, PSEN2, presenilin 1 and 2; APP, amyloid precursor protein; $A \beta$, amyloid $\beta$ peptide; APOE, apolipoprotein e; SORL1, sortilin-related receptor 1; BDNF, brain-derived neurotrophic factor; 3D, three-dimensional; NFTs, neurofibrillary tangles

\section{Introduction}

Since hESCs were discovered many advances have been taken place in the stem cells field. ${ }^{1}$ A major breakthrough in this arena came ten years ago, when the Yamanaka's lab generated iPSCs from mouse and human fibroblasts by using a cocktail of transcription factors. ${ }^{2,3}$ These cells were similar to ESCs in morphology, gene expression profile and differentiation potential ${ }^{4}$ but, without the ethical concerns and technical constraints hESCs entail because they have an adult somatic origin. iPSCs significance is apparent in clinical application, as these cells can avoid immune rejection because they can be derived from the patient's own cells. One step forward in stem cell technology is what has been called direct reprogramming, that is, the ability to fully differentiate cells and induce them toward other cell types while circumventing an intermediate pluripotent state. It has been reported the generation of iNSCs from mouse and human fibroblasts by direct reprogramming with a single factor, Sox $2 .{ }^{5}$ These iNSCs express NSCs markers and resemble wild-type NSCs. ${ }^{6,7}$ Thus, self-renewable and multipotent iNSCs can be generated directly from fibroblasts by reprogramming. However, the need for using transgenic reprogramming factors in this approach still presents major technical and safety concerns. ${ }^{8}$ In this sense the use of small molecules seems to overcome those technical drawbacks being capable of converting human fibroblasts into neurons with a cocktail of small molecules supplemented with chemicals known to promote the differentiation of neural progenitors into neurons. ${ }^{9}$ iNs produced by this cocktail possess typical functional hallmarks of neurons. ${ }^{10}$ Recently, the application of stem cell research to neurodegenerative disorders is rapidly increasing, not only for cell replacement therapy ${ }^{11}$ but also as potential models of the malady in vitro ${ }^{12,13}$ contributing to improve knowledge of the cellular and molecular mechanisms of neurodegeneration and innovative drug discovery such as AD. The main goal of this mini-review is to show current progress in the field of iPSCs, and how these new approaches are creating innovative AD disease models which subsequently will contribute to elucidate its pathogenesis and future cure.

\section{Discussion}

$\mathrm{AD}$ is the most frequent form of dementia in which the major risk factor is senescence, especially after the age of 65 . Nowadays, ordinary treatments cannot stop or reverse its advance, but some symptoms may momentarily be reduced. The most common type of $\mathrm{AD}$ is sporadic which accounts for $95 \%$ cases in contrast with less than $5 \%$ for familial. ${ }^{14}$ Indistinctly to $\mathrm{AD}$ form, these patients exhibit progressive dementia with anatomically extracellular $A \beta$ plaques, and intracellular NFTs as a result of hyperphosphorylated tau protein accumulation. In both types, they share the same clinical and pathological process in a way that the imbalance between production 
and degradation of $\mathrm{A} \beta$ results in neuron loss. Given that $\mathrm{AD}$ is a heterogenous disorder with multiple causes that can be involved in neurodegeneration, its final etiology is not thoroughly understood. However, genetic factor is considered to be among main contributors to the risk of AD. Mutations in disease-causing genes and diseaserisk genes have been identified and linked with FAD and SAD. ${ }^{15}$ Prominently inherited mutations of PSEN1, PSEN2 genes as well as their substrate APP, whose excision give rise to $\mathrm{A} \beta$ peptides depending on intervening secretases are among the principal causes of earlyonset FAD. ${ }^{14-17}$ However, the etiology of the most abundant form of $\mathrm{AD}$, sporadic, is not yet well known since it depends on a sort variety of circumstances such as gender, age, sociocultural environment and mutations in $\mathrm{APOE}^{15,18}$ whose frequent variations are the only well documented association with SAD. The boundaries for AD pathogenesis knowledge are, on the one hand, the difficulties to obtain live neurons from patients, since samples come from post-mortem tissue. On the other hand, the conventional experimental models with animals or cell lines are quite inappropriate for that disorder, since it is difficult to replicate the disease traits in a lab setting. ${ }^{19}$ Therefore; $\mathrm{AD}$ models based in iPSC are suitable for this purpose. To study FAD pathogenesis, iPSCs-derived neurons have been used from patients with mutations in APP, PSEN1, and PSEN2 in which the production of $\mathrm{A} \beta$ peptides and the accumulation of phosphorylated tau protein was analyzed. Different studies have showed that the expression of $A \beta$ peptides - $A \beta 38, A \beta 40, A \beta 42-$ and ratio among them was altered compared to iPSCs from healthy donors revealing the importance that balance may play for $\mathrm{AD}$ development. On the contrary, no accumulation of tau protein was observed in this type of FADderived neurons, revealing that at least for that genotype this protein may not be the main cause of ailment. ${ }^{12,15,20}$ Indeed, some studies have showed that iNs and iPSCs-derived neurons from FAD patient fibroblasts had an increased $A \beta 42 / A \beta 40$ ratio that resembles that observed in AD brains. ${ }^{10,21,22}$ Similarly, human iNs from familial AD patients recapitulated the pathology of altered processing and aberrant endosomal localization of APP as well as increased production of A $\beta$ peptides. ${ }^{23}$ The iPSCs technique is especially useful for FAD but it is more so in the case of the sporadic form whose low prevalence makes it difficult to identify the agent responsible of the disorder. Therefore, the underlying molecular events can be approached without intrusion of external factors, allowing the identification of new networks of regulated genes related to $\mathrm{AD}$ progress. Thus, based in this technology many studies have revealed that numerous other genes can become susceptible in the sporadic form. One of those genes is the aforementioned APOE gene, responsible of lipids transportation through the lymphatic and circulatory systems, which suggests that lipid metabolism plays a role in the etiology of AD. ${ }^{24}$ Similarly, there is a significant correlation between expression of SORL1 haplotype which encodes an endocytic trafficking factor whose levels modulate the processing of APP to A $\beta$ as well as other proteolytic products implicated in SAD, and the BDNF gene. ${ }^{25,26}$ Because of the varied nature of SAD phenotype iPSCs-derived neurons from those patients without inheritance must be extensively expanded to obtain statistically significant data. Moreover, detection of slight phenotypic changes between cells differentiated from a patient or control iPSCs may not reveal a relevant phenotypic difference between both groups of cells but rather reflect the system's intrinsic dissimilarity between individual iPSC lines. ${ }^{27}$ It is for that reason that isogenic pairs composed of disease-specific and control iPSCs that differ exclusively at the disease-causing mutation have been generated, being presently the best disease in a dish model strategy. ${ }^{28,29}$ This approach has been possible thanks to last advances in gene editing (CRISPR-Cas9; TALENs; ZFN). ${ }^{30,31}$ iPSCs with heterozygous and homozygous mutations in the genes for APP and PSEN1 generated through CRISPR/ Cas9 technology and differentiated into cortical neurons have recapitulated specific features of $\mathrm{AD} .{ }^{32}$ Another important achievement for disease modeling is the recent advances in 3D cell culture Matrigel-based technology. This system has made it possible to generate novel 3D neural cell culture models capable to recapitulate $\mathrm{AD}$ associated traits seen in post-mortem samples such as $A \beta$ deposition similar to amyloid plaques, and NFT-like tau aggregates from patient iPSC-derived neurons or from genetically modified human stem cell lines; this pattern has not been feasible to be reproduced even in animal models. ${ }^{33}$ These results reveal that iPSCs based technology together with 3D human cell culture models and genome editing are very promising tools to study human brain-like environment and open a new dimension for regenerative medicine, disease modeling and high-throughput drug screening to treat $\mathrm{AD}{ }^{34,35}$

\section{Conclusion}

Although $\mathrm{AD}$ was first described more than a century ago, and it is the most prevalent neurodegenerative disease worldwide, the cause of this disorder remains unclear. Many efforts in favor of revealing its etiology have been made along these years but conventional experimental models have not been able to unravel its pathogenesis. A new era in regenerative medicine arose when iPSCs were discovered. iPSCs are similar to ESCs but without the ethical constraints related to embryo destruction, and they can be obtained without depending upon the naturally limited harvest of ESCs. Considering that iPSCs come from adult somatic cells and given that they can be derived to any cell type, such as neurons, iPSCs have become a perfect tool to develop new disease in a dish models of AD. Furthermore, the iPSCs technology offers new possibilities for biomedical research and clinical applications, not only for in vitro cellular models of neurodegenerative diseases but for autologous transplantation for which theoretically no immunosuppressive therapy would be necessary. However, many limitations still affect the application of this technology to personalized medicine in a clinical setting. The main restraints are related to technical issues, such as the development of safe and efficient methods for iPSCs generation. Additionally, not only iPSCs are expected to present the same challenges as ESCs but they also bear the same genetic traits as the patient, and the particulars of the reprogramming process may give rise to further problems. Another important issue is the risk of tumor formation by iPSCs, which is even higher than that of ESCs, especially when viral or genome-integrating vectors are used. Currently, the adoption of disease models based on 3D cell culture technology will be critical to recreate functional brain structures with multiple cell types, more similar anatomically to AD affected brain than conventional cell culture models. Finally, genome editing has proven to be a very powerful tool for modifying a DNA sequence that along with iPSCs technology will bring in the near future new experimental models of disease, and encouraging prospects for gene therapy directed at human diseases treatment.

\section{Acknowledgements}

This study was supported by grant from MINECO-Retos Colaboración 2015 (RTC-2015-3805-1).

\section{Conflict of interest}

The author declares no conflict of interest. 


\section{References}

1. Thomson JA, Itskoviz Eldor J, Shapiro SS, et al. Embryonic stem cell lines derived from human blastocysts. Science. 1998;282(5391):11451147

2. Takahashi K, Yamanaka S. Induction of pluripotent stem cells from mouse embryonic and adult fibroblast cultures by defined factors. Cell. 2006;126(4):663-676.

3. Takahashi K, Tanabe K, Ohnuki M, et al. Induction of pluripotent stem cells from adult human fibroblasts by defined factors. Cell. 2007;131(5):861-872.

4. Phanstiel DH, Brumbaugh J, Wenger CD, et al. Proteomic and phosphoproteomic comparison of human ES and iPS cells. Nat Methods. 2011;8(10):821-827.

5. Ring KL, Tong LM, Balestra ME, et al. Direct reprogramming of mouse and human fibroblasts into multipotent neural stem cells with a single factor. Cell Stem Cell. 2012;11(1):100-109.

6. Martínez Morales PL, Liste I. Stem cells as in vitro model of Parkinson's disease. Stem Cells Int. 2012;2012:980941.

7. Durnaoglu S, Genc S, Genc K. Patient-specific pluripotent stem cells in neurological diseases. Stem Cells Int. 2011;2011:212487.

8. Revilla A, González C, Iriondo A, et al. Current advances in the generation of human iPS cells: implications in cell-based regenerative medicine. J Tissue Eng Regen Med. 2016;10(11):893-907.

9. Cheng L, Hu W, Qiu B, et al. Generation of neural progenitor cells by chemical cocktails and hypoxia. Cell Res. 2014;24(6):665-679.

10. Hu W, Qiu B, Guan W, et al. Direct conversion of normal and Alzheimer's disease human fibroblasts into neuronal cells by small molecules. Cell Stem Cell. 2015;17(2):204-212.

11. Liu X, Li F, Stubblefield EA, et al. Direct reprogramming of human fibroblasts into dopaminergic neuron-like cells. Cell Res. 2012;22(2):321-332.

12. Israel MA, Yuan SH, Bardy C, et al. Probing sporadic and familial Alzheimer's disease using induced pluripotent stem cells. Nature. 2012;482(7384):216-220

13. Abdullah AI, Pollock A, Sun T. The path from skin to brain: generation of functional neurons from fibroblasts. Mol Neurobiol. 2012;45(3):586595.

14. Hardy J, Gwinn Hardy K. Genetic classification of primary neurodegenerative disease. Science. 1998;282(5391):1075-1079.

15. Zhang W, Jiao B, Zhou M, et al. Modeling Alzheimer's disease with induced pluripotent stem cells: current challenges and future concerns. Stem Cells Int. 2016;2016:7828049.

16. Soldner F, Jaenisch R. Dissecting risk haplotypes in sporadic Alzheimer's disease. Cell Stem Cell. 2015;16(4):341-342.

17. Campion D, Dumanchin C, Hannequin D, et al. Early-onset autosomal dominant Alzheimer disease: prevalence, genetic heterogeneity, and mutation spectrum. Am J Hum Genet. 1999;3:664-670.

18. Borenstein AR, Copenhaver CI, Mortimer JA. Early-life risk factors for Alzheimer disease. Alzheimer Dis Assoc Disord. 2006;20(1):63-72.
19. Wan W, Cao L, Kalionis B, et al. Applications of induced pluripotent stem cells in studying the neurodegenerative diseases. Stem Cells Int. 2015;2015:382530.

20. Yagi T, Ito D, Okada Y, et al. Modeling familial Alzheimer's disease with induced pluripotent stem cells. Hum Mol Genet. 2011;20(23):4530 4539 .

21. Sproul AA, Jacob S, Pre D, et al. Characterization and molecular profiling of PSEN1 familial Alzheimer's disease iPSC-derived neural progenitors. PLoS One. 2014;9(1):e84547.

22. Babos K, Ichida JK. Small molecules take a big step by converting fibroblasts into neurons. Cell Stem Cell. 2015;17(2):127-129.

23. Young JE, Goldstein LSB. Alzheimer's disease in a dish: promises and challenges of human stem cell models. Hum Mol Genet. 2012;21(R1):R82-R89.

24. Wojda U, Kuznicki J. Alzheimer's disease modeling: Ups, downs, and perspectives for human induced pluripotent stem cells. Journal of Alzheimer's Disease. 2013;34(3):563-88.

25. Young JE, Boulanger Weill J, Williams DA, et al. Elucidating molecular phenotypes caused by the SOR1 Alzheimer's disease genetic risk factor using human induced pluripotent stem cells. Cell Stem Cell. 2015;16(4):373-385.

26. Devineni A, Tohme S, Kody MT, et al. Stepping back to move forward: a current review of iPSCs in the fight against Alzheimer's disease. Am J Stem Cells. 2016;5(3):99-106.

27. Soldner F, Jaenisch R. Medicine. iPSC disease modeling. Science. 2012;338:1155-1156.

28. Soldner F, Laganière $\mathrm{J}$, Cheng $\mathrm{AW}$, et al. Generation of isogenic pluripotent stem cells differing exclusively at two early onset Parkinson point mutations. Cell. 2011;146(2):318-331.

29. Hockemeyer D, Jaenisch R. Induced pluripotent stem cells meet genome editing. Cell Stem Cell. 2016;18(5):573-586.

30. Hendriks WT, Warren CR, Cowan CA. Genome editing in human pluripotent stem cells: approaches, pitfalls, and solutions. Cell Stem Cell. 2016;18(1):53-65.

31. Kimbrel EA, Lanza R. Pluripotent stem cells: the last 10 years. Regen Med. 2016;11(8):831-847.

32. Paquet D, Kwart D, Chen A, et al. Efficient introduction of specific homozygous and heterozygous mutations using CRISPR/Cas9. Nature. 2016;533(7601):125-129.

33. Zhang D, Pekkanen Mattila M, Shahsavani M, et al. A 3D Alzheimer's disease culture model and the induction of P21-activated kinase mediated sensing in iPSC derived neurons. Biomaterials. 2014;35(5):1420-1428.

34. Choi SH, Kim YH, Quinti L, et al. 3D culture models of Alzheimer's disease: a road map to a "cure-in-a-dish. Mol Neurodegener. 2016;11(1):75.

35. Peng X, Xing P, Li X, et al. Towards Personalized Intervention for Alzheimer's Disease. Genomics, Proteomics and Bioinformatics. 2016;14(5):289-297. 